International Journal of Engineering \& Technology, $7(2.12)(2018) 329-332$
International Journal of Engineering \& Technology
Website: $w$ ww.sciencepubco.com/index.php/IJET
Research Paper

\title{
IOT based modeling of closed transition transfer switch in IEC 61850
}

\author{
Hyun Sung Lim ${ }^{1}$, In Ho Ryu ${ }^{2}$, Jun Ho Bang ${ }^{2}$ \\ ${ }^{1}$ Department of Future Technology Research, Electrical Safety Institute, Wanju, 55365, KOREA \\ ${ }^{2}$ Department of IT Applied System Engineering, UNIV of CHONBUK, Jeonju, 54896, KOREA \\ *Corresponding author E-mail: hslim@kesco.or.kr
}

\begin{abstract}
Background/Objectives: The power demand of utility electrical power has stimulated the use of distributed energy for peak shaving. Distributed energy resources need to be monitored and controlled like Internet of Things.

Methods/Statistical analysis: Distributed Energy Resources (DERs) are small scale electric energy system like Micro Turbine, Photovoltaic, Wind power, Small Generator and widely spread in Korea. For utilizing electrical device with Internet of Things, we need integrated system and adapt International Electrical Code like IEC 61850.

Findings: To connect DERs with uninterrupted grid system, it required Closed Transition Transfer Switch (CTTS). Existing International Electrical Code presented some distributed energy resource by IEC 61850-7-420. However, the switch like CTTS is not presented. So, we described modeling of CTTS in IEC 61850 and verified monitoring data with TCP/IP.

Improvements/Applications: The proposed modeling of CTTS not only combines the DERs with grid system but also conjugates smart grid system with IOT Technology.
\end{abstract}

Keywords: IoT; Closed Transition Transfer Switch; IEC 61850; Smart Grid; Uninterrupted System

\section{Introduction}

Recently, peak power demand has been renewed to the maximum and backup power has dropped to a critical level, aggravating power supply-demand situation such as rolling blackouts ${ }^{1}$. To solve the problems the usage of Distributed Energy Resource (DER) which are connected with grid $^{2}$. DER is a small power system like Micro Turbine, Photovoltaic, Wind power system, small generator. Each of DERs has own standard by manufactures. Integration of DERs such as wind, energy storage, and DR to existing electrical networks can help companies eliminate the investment cost related to transmission line expansion and establishment of new conventional power plants ${ }^{3}$. To accommodate toe DERs into the existing system, it is required in uninterrupted connection technology like Closed Transition Transfer Switch (CTTS). Existing connection system of Automatic Transfer Switch (ATS) makes interruption because the system has single switch. In order to solve the problem, CTTS is composed two switches for each power source. The advantages are that DERs can be operated without interrupting power to loads and power can be retransferred to the utility after a failure without interrupting power to loads. The system will be connected with various DERs and required a flexible and reliable communication protocol for control and monitor functions of CTTS. Micro gird system has many power source and needs integrated standardization for Internet of Things. IEC 61850 is standard communication of power system and integrated devices of different types into the system. IEC $61850-7-420$ is committed to the principle of standards to support the various DERs ${ }^{4}$. However, interconnection for DERs with switch has not been presented. So, this paper describes the configuration and function of CTTS for mapping to IEC 61850 and considers existing logical nodes and uses new logical nodes (LNs).

\subsection{Technical background}

The CTTS consists of two switches for the grid and generator as shown in Figure 1.
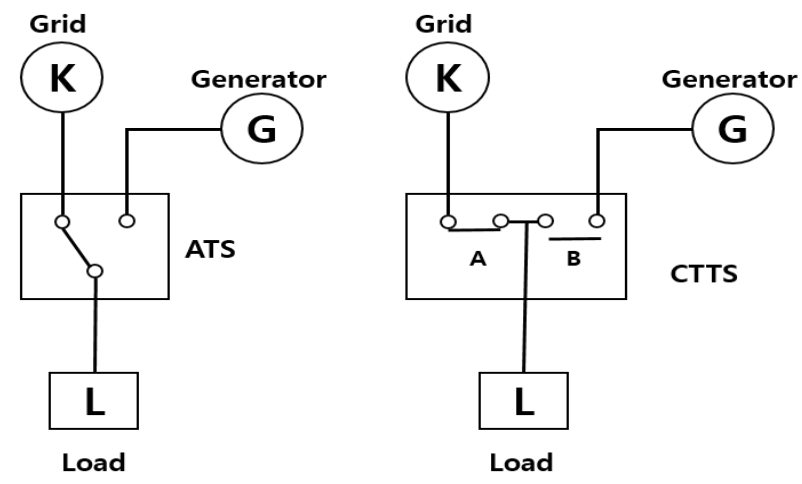

Fig. 1: Diagram of ATS \& CTTS.

If synchronization conditions with grid power is satisfied after generator is activated, it is transferred to the generator power after parallel operation within $100 \mathrm{~ms}^{5}$. The CTTS synchronization conditions are designed as stated in Table 1. 
Table 1: Synchronization Condition \& Parallel Operation Times

\begin{tabular}{ll}
\hline Category & Conditions \\
\hline Voltage Phase Difference & $<10^{\circ}$ \\
Frequency Difference & $<1 \mathrm{~Hz}$ \\
Voltage Difference & $<5 \%$ \\
Parallel Operation Times & $<100 \mathrm{~ms}$ \\
\hline
\end{tabular}

CTTS is generally utilized for interconnected reciprocating engine system like emergency generator in Smart grid system. According to the Korea Electrical Safety Corporation's statistics dated June 2013 , the country had 69,986 emergency generator with a total capacity of $21 \mathrm{GW}$, which is equivalent to the capacity of approximately 20 nuclear power generating unit. According to Table 2 below, operating hours vary depending on the ratio of investment cost. Despite the support (3 years, 100\%), if an emergency generator is operated for more than 70.9 hours per years, it is more efficient than Demand Resource Market operating costs. If business models beneficial to customers are developed and grid-connection technology is secured, it would be a very effective demand resources [6].

Table 2: Emergency Generator Required Operating Hours Equal to the Operating Cost of the DRM

\begin{tabular}{llll}
\hline \multirow{2}{*}{$\begin{array}{l}\text { Investment Sup- } \\
\text { port Ratio }\end{array}$} & \multicolumn{4}{l}{ Compulsory Participation Commitment Period for } \\
& $\begin{array}{l}\text { Demand Management } \\
3 \text { year }\end{array}$ & 5 year & 10 year \\
\hline $50 \%$ & $35.4 \mathrm{~h} /$ year & $22.5 \mathrm{~h} /$ year & $13.0 \mathrm{~h} /$ year \\
$70 \%$ & $49.6 \mathrm{~h} /$ year & $31.6 \mathrm{~h} /$ year & $18.2 \mathrm{~h} /$ year \\
$100 \%$ & $70.9 \mathrm{~h} /$ year & $45.1 \mathrm{~h} /$ year & $26.1 \mathrm{~h} /$ year \\
\hline
\end{tabular}

Therefore some of emergency generator utilized as demand resources, they might significantly decrease peak power. For utilizing emergency generator, the system must be uninterrupted status So, it required Closed Transition Transfer Switch and International Standardization for making Smart grid system based on IoT technology

\subsection{Proposed modelling of CTTS}

The proposed modeling of CTTS described International Electrotechnical Commission Standards. The international standard IEC 61850 has been recognized as a globally accepted solution enabling integration of heterogeneous devices and applications in the power system automation domain ${ }^{7}$. So we will describe CTTS with IEC 61850 standards using existing Logical Nodes and new Logical Nodes.

\subsection{Organization of the paper}

Section 2 define mapping CTTS data classes and attributes. Section 3and Section 4 are the hardware \& software implementation detail. Section 5 compare between CTTS Modbus data and Mapping data in IEC 61850. Section 6 concludes the paper.

\section{CTTS mapping to IEC 61850}

IEC 61850 allow to user can use the modeling method and Common Data Classes (CDC) in IEC 61850-7-1. The description of each logical nodes is Figure 2 as follows

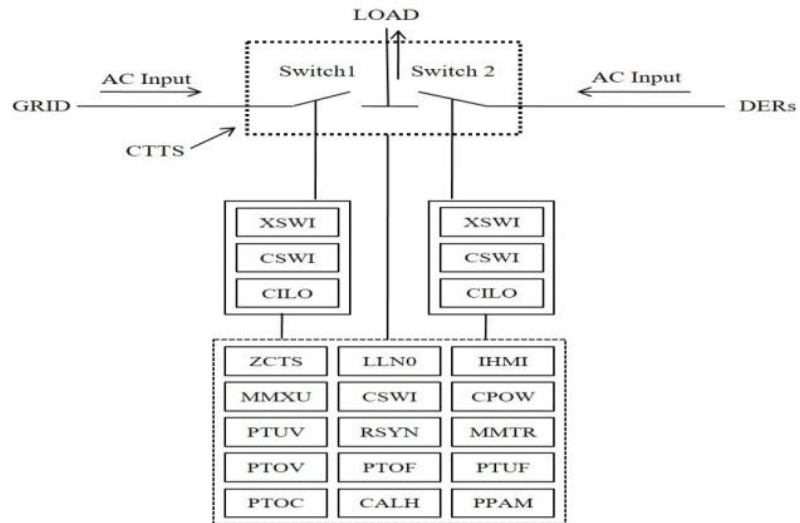

Fig. 2: CTTS Mapping to IEC 61850.

1) ZCTS is the logical node (LN) defines the characteristics of the closed switch in CTTS. There is new logical node ZCTS which is inherited from logical node class, $\mathrm{Z}$, denotes to the $\mathrm{LN}$ group for further equipment. $\mathrm{C}$ is meaning of Closed and TS is Transfer Switch.

2) LLNO logical node device for CTTS that includes common information for logical device.

3) MMXU \& MMTR are Grid and DERs values at CTTS

4) PTUV (Under Voltage), PTOV (Over Voltage), PTOF(Over Frequency), PTUF(Under Frequency), PTOC(Over Current), PPAM(Phase Angle) are protection logical nodes.

5) CPOW is the point-on -wave breaker controller LN provides all functionality to close or open a circuit breaker at a certain instant of time.

6) CSWI is the switch control LN handles all switchgear operations from the operators and from related automatics.

7) IHMI is front panel operator interface at bay level to be used for configuration, i.e. local control

8) RSYN is a synchronizing function that produces a release for a closing command of circuit breaker between two circuits whose voltages are within prescribed limits of magnitude, phase angle, and frequency.

Table 3 shows the new proposed LN to characterize a closed transition transfer switch. The letter "T" is Transient data object. The status of data objects with this designation is momentary and must be logged or reported to provide evidence of their momentary state. The column specified with $\mathrm{M} / \mathrm{O} / \mathrm{C}$ defines whether data objects are mandatory $(\mathrm{M})$ or optional $(\mathrm{O})$ or conditional $(\mathrm{C})$ for the instantiation of a specific logical node 4 .

Table3: Ctts Zcts Class

\begin{tabular}{|c|c|c|c|c|}
\hline \multirow{2}{*}{\multicolumn{5}{|c|}{ ZCTS class }} \\
\hline & & & & \\
\hline $\begin{array}{l}\text { Data object } \\
\text { name }\end{array}$ & $\begin{array}{l}\text { Common } \\
\text { data class }\end{array}$ & Explanation & $\mathrm{T}$ & $\mathrm{M} / \mathrm{O} / \mathrm{C}$ \\
\hline \multicolumn{5}{|c|}{ Measured values } \\
\hline DERtyp & ING & Type of DER Unit & & M \\
\hline OpTmh & INS & Operation time & & M \\
\hline AutoManCtl & SPC & $\begin{array}{l}\text { Automatic or Manual } \\
\text { Mode }\end{array}$ & & M \\
\hline FltAck & SPC & $\begin{array}{l}\text { Acknowledge fault } \\
\text { clearing }\end{array}$ & & $\mathrm{O}$ \\
\hline GnSync & SPS & $\begin{array}{l}\text { Generator is synchro- } \\
\text { nized }\end{array}$ & & $\mathrm{O}$ \\
\hline AVR & MV & $\begin{array}{l}\text { Automatic Voltage } \\
\text { Regulator }\end{array}$ & & $\mathrm{O}$ \\
\hline GnCtl & DPC & $\begin{array}{l}\text { Starts or stops the gen- } \\
\text { erator }\end{array}$ & & $\mathrm{O}$ \\
\hline HzRtg & ASG & Nominal frequency & & $\mathrm{O}$ \\
\hline VRtg & ASG & Voltage level rating & & $\mathrm{O}$ \\
\hline ARtg & ASG & $\begin{array}{l}\text { Current rating under } \\
\text { nominal voltage }\end{array}$ & & $\mathrm{O}$ \\
\hline
\end{tabular}

\section{Hardware implementation}

CTTS internal interconnection used CAN communication and output data used RS 485 connection. And the data type of CTTS 
output is Modbus serial communication protocol. Modbus enables communication among many devices connected to the same network. To convert Modus data to IEC 61850, we installed gateway. The IEC 61850 gateway has ARM Cortex M5 Series 500Mhz CPU, 512MB Ram, 512MB, Flash memory. It supported IEC 61840 Group 1 MMS protocol and RS 485 based 2 Channel communication (Half Duplex Mode). Main VPN has Quad Core CPU, 4GB memory, dual redundant power supply system. It supported standard IPSec Protocol, IKE V1, V2, IKE Diffie-Helman Group $1,2,5,14,15,16,17,18$, Bandwidth-based load-balancing. Figure 3 displays the hardware implementation.

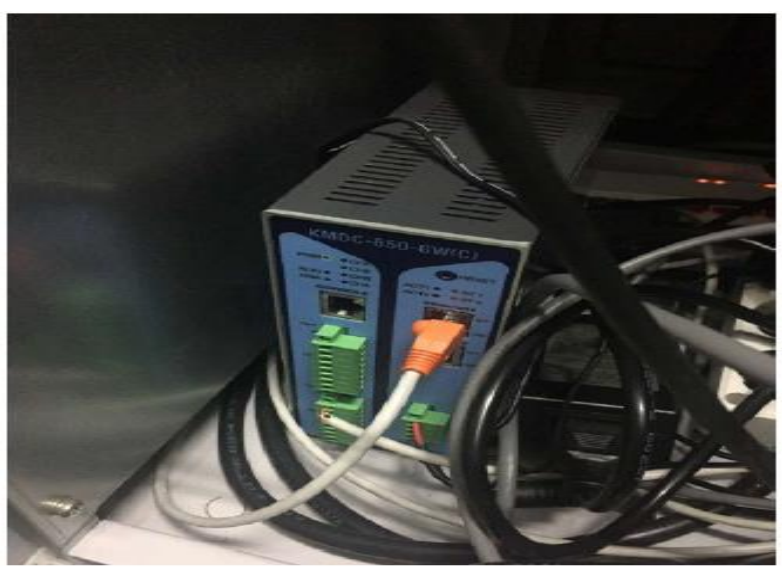

Fig. 3: Hardware Implementation.

\section{Software implementation}

Modeling of CTTS started to make a modeling Markup language based on XML considering Intelligent Electrical Devices characteristic and attributes. This modeling define IEC 61850 Class and FC details. We use the SCL Forge software and verified standard compliance.

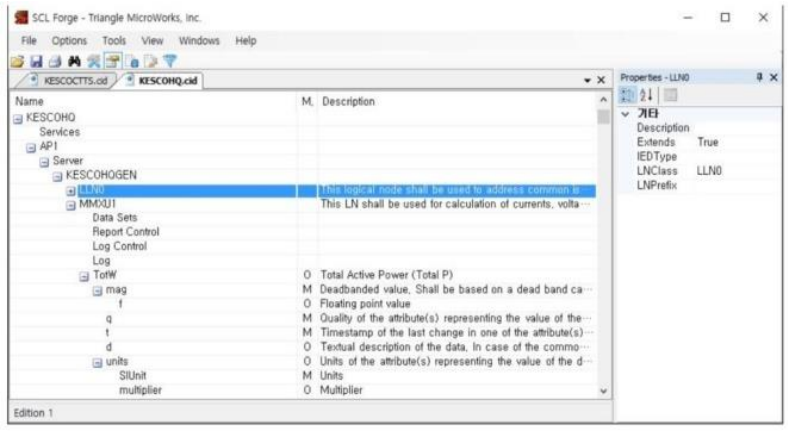

Fig. 4: IED Modeling of SCL Tool (SCL Forge).

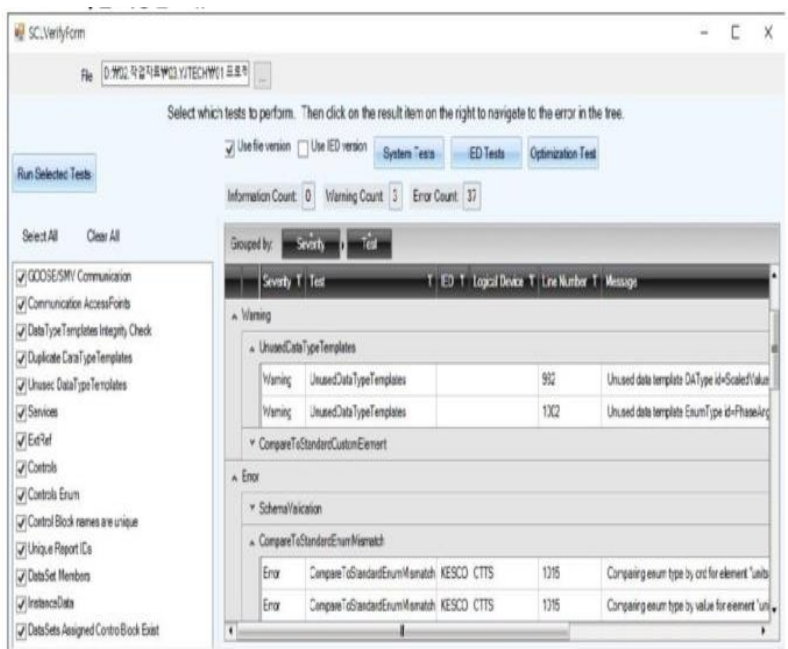

Fig. 5: SCL Verify Error Check.
Figure 6 is Modbus data of Closed Transition Transfer Switch connected by generator and Figure 7 converts to IEC 61850 data format from Modbus data.

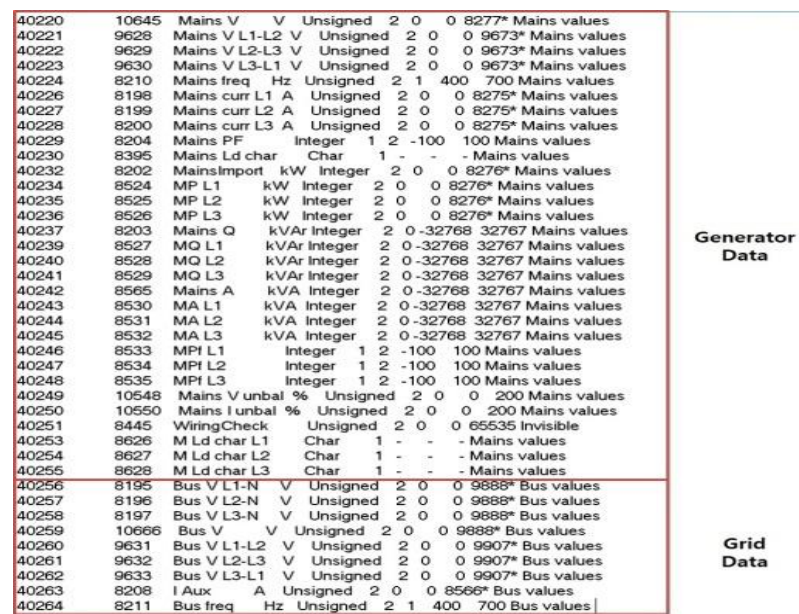

Fig. 6: Modbus Address of CTTS.

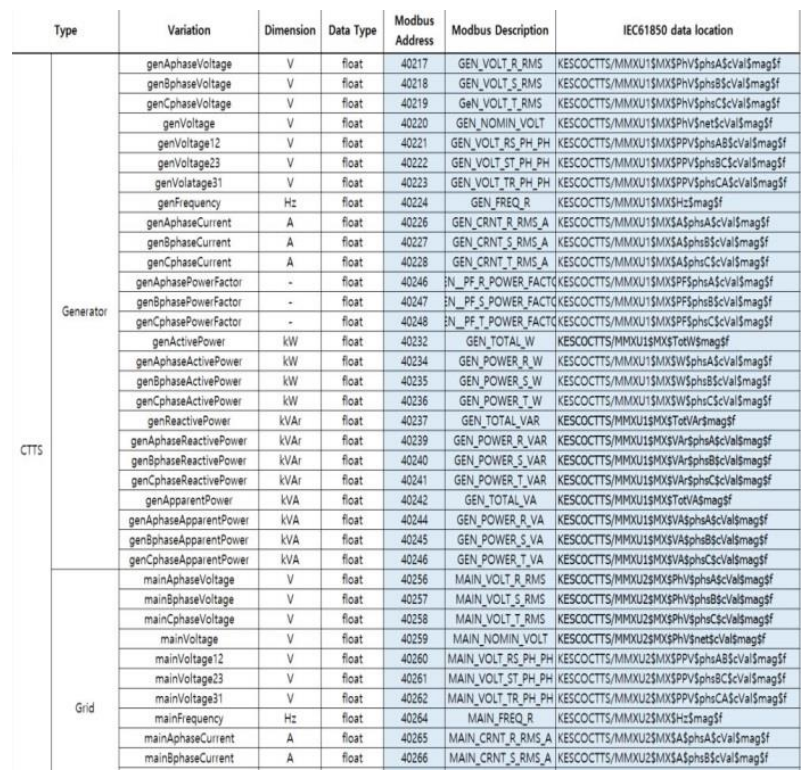

Fig. 7: IEC 61850 Data.

\section{Results}

Figure 8 represents accurately convert an existing communication protocol (Modbus) and IEC 61850. The Grid Phase to Voltage show 217V (Phase A), 216V (Phase B), 217V (Phase C) and Voltage to Voltage is $380 \mathrm{~V}$. And The Grid Frequency is $59.9 \mathrm{~Hz}$.

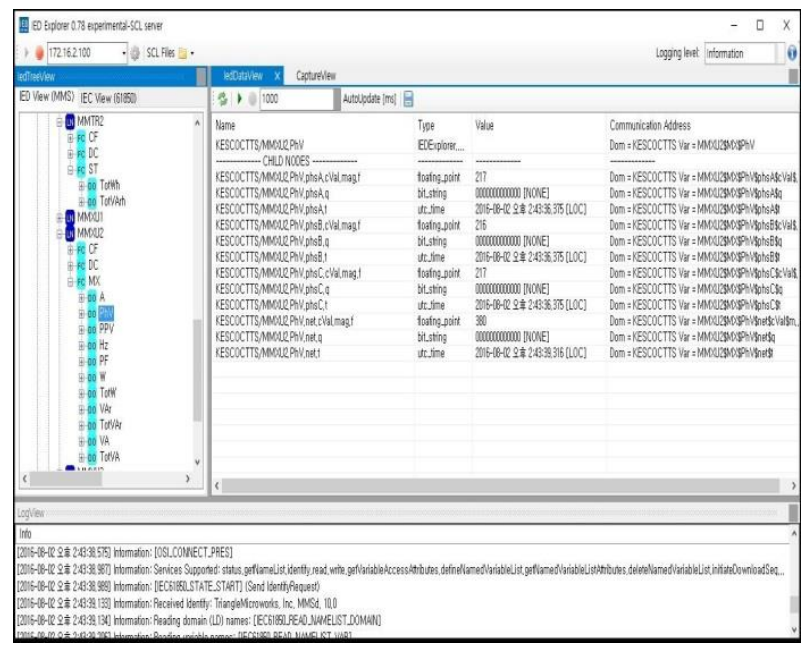




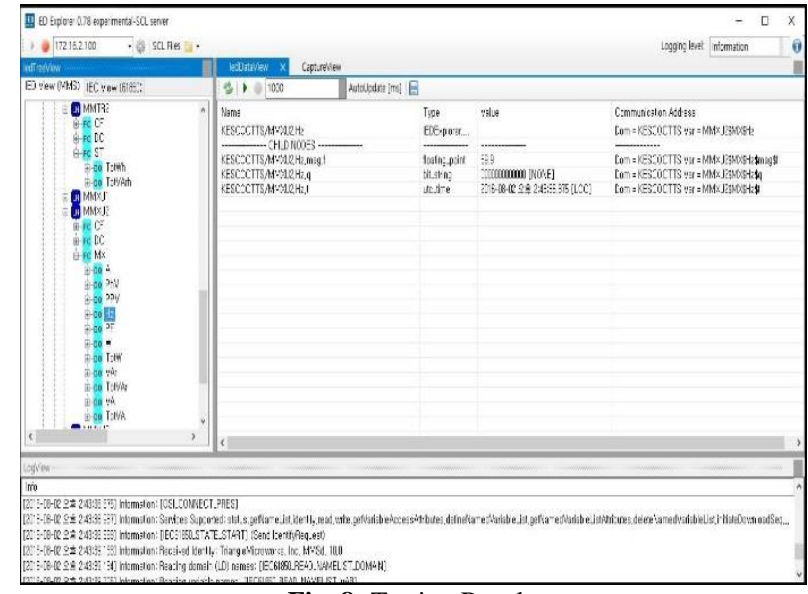

Fig. 8: Testing Result.

\section{Conclusion}

This paper proposed mapping CTTS to IEC 61850 for using IoT Technology. First we defined the CTTS data classes and attributes. We used new logical node like ZCTS because there is not defined switch system in IEC 61850 standard. The CTTS system support output data in RS485 and the data type is Modbus data. So, we convert to the IEC 61850 standard data type from Modbus data. And then the comparison data has same result with onsite and remote site. Therefore, the proposed scheme can be applied to remote system like IoT.

\section{References}

[1] Putta Sindhuja, M.S. Balamurugan, Smart Power Monitoring and Control System through Internet of tings using Cloud Data Storage, Aug 2015, 8(19).

[2] Huang, A.Q., The future renewable electric energy delivery and management(FREEDM) system, IEEE, 2011, 99, pp.133-148

[3] Samaneh Pazouki, Shahab Ardalan, Mahmoud-Reza Haghifam, Demand Response Programs in Optimal Operation of Multi-Carrier Energy Networks, Indian Journal of Science and Technology, Dec 2015, 8(33).

[4] IEC, Communication networks and systems in substation Part 7 420: Basic communication structure, 2011 UL, UL 1008: Transfer Switch Equipment, 2014.

[5] J K Choi, J H Jung, J H Lim, S S Ma, K J Park, A Study on Utilization of Backup Generators for Demand Side Management, KIEE, May 2012.

[6] Salvatore Cavalieri, Alessio Regalbuto, Mapping IEC $61850 \mathrm{SCl}$ to IPC UA for Smart Grid Application, IEEE, 2015 nurses in korea. Diabetes Research and Clinical Practice 77, 199-204.

[7] McMahon GT, Gomes HE, Hohne SH, Hu TM, Levine BA \& Conlin PR (2005), Web-based care management in patients with poorly controlled diabetes. Diabetes Care 28, 1624-1629.

[8] Thakurdesai PA, Kole PL \& Pareek RP (2004), Evaluation of the quality and contents of diabetes mellitus patient education on Internet. Patient Education and Counseling 53, 309-313. 